\title{
»Mama, sing mir mal das Buch!«. Einige Überlegungen zu ‘guten` prosodischen Gestalten im frühen Spracherwerb
}

In den letzten 30 Jahren hat die Forschung zum Spracherwerb erstaunliche Erkenntnisse darüber hervorgebracht, wie Kleinkinder ihre Muttersprache lernen. Dies geschieht ausgesprochen schnell und effektiv: Bereits nach den ersten drei Lebensjahren werden sie zu ausreichend kompetenten Hörern und Benutzern ihrer Muttersprache. Dies ist deshalb möglich, da Kinder schon ab dem ersten Tag ausgeprägte Mustererkenner sind, d.h. ihre Wahrnehmung (und auch Aufmerksamkeit) scheint sich auf solche Merkmale des sprachlichen Inputs zu richten, die zum einen regelhaft wiederkehren und daher statistisch wahrscheinlich sind und zum anderen Gestalten bilden, die auffällig sowie gut zu merken sind. In diesem Beitrag möchte ich an Beispielen aus eigener Feldforschung solche Gestalten aufzeigen, die in der lautlichen Form sowohl gesprochener als auch gesungener Sprache mit Kleinkindern enthalten sind und ihren Wert für die Sprachentwicklung diskutieren. Im Mittelpunkt der Überlegungen steht ein Prozess, der hier als Optimierung poetischer Struktur (im Sinne Jakobsons 1960) bezeichnet wird. Unter Rückgriff auf gestaltpsychologische Ansätze wird es möglich, das Kontinuum Sprache - poetische Sprache - Gesang einheitlich zu betrachten und neue Fragestellungen für die Forschung zum frühen Spracherwerb und zu Eltern-Kind-Interaktionen aufzuzeigen.

\section{Prosodic Bootstrapping: Sprachaneignung dank lautlicher Struktur}

Die lautlich-klangliche Form eines Sprachsignals kodiert viele Informationen über phonologische, aber auch morpho-syntaktische und semantisch-pragmatische Einheiten der Sprache. Wie z. B. Selkirk (1980) oder Nespor/Vogel (1986) zeigten, existieren Zuordnungs-Regeln, die phonologische, insbesondere prosodische ${ }^{1}$ Domänen und Konstituenten auf verschiedene lexikalisch-grammatische Domänen

${ }^{1}$ Zur Prosodie zählen alle phonologisch relevanten Schalleigenschaften, die über den Einzellaut hinausgehen. Als prosodisch gelten demnach Phänomene und Strukturen wie z. B. Silben, Akzente, Ton, Intonation. 
und Konstituenten abbilden. So gibt es Sprachen wie das Polnische, dessen Wortakzent zuverlässig auf der vorletzten Silbe eines Wortes auftritt und damit die rechte Grenze eines Wortes markiert. In Sprachen, die ein Intonationssystem haben (z. B. das Englische, Deutsche) signalisieren bestimmte tonale Bewegungen die Grenzen einer Phrase, die gut mit syntaktischen Großabschnitten zusammenfallen. Eine Spracherwerbshypothese, die Prosodic Bootstrapping genannt wird (basierend auf Gleitman/Wanner 1982), geht davon aus, dass Kinder dank dieser regelhaften Eigenschaften des Sprachsignals zur abstrakteren grammatischen Form der Sprache finden können. Diese These wurde seitdem vielfach getestet und bestätigt. HirshPasek et al. (1987) beispielsweise fanden, dass sieben bis zehn Monate alte englischlernende Kinder Texte mit Pausen an Satz-/Phrasengrenzen bevorzugten und folgerten, dass sie in der Lage wären, sinnvolle Einheiten aus dem Lautstrom zu segmentieren. Schon jüngere Kinder reagieren sehr sensibel auf die globalen Intonationskonturen von Sprache (z. B. Mandel et al. 1996) und die globale rhythmische Form (z. B. Mehler et al. 1988, Überblick in Nazzi/Ramus 2003). In mehreren Studien (z. B. Morgan 1996, Echols/Crowhurst/Childers 1997; Jusczyk/Houston/ Newsome 1999; Echols 2000) zeigte sich, dass englischlernende Kinder ab ca. sieben Monaten ein trochäisches Betonungsmuster nutzen, um zweisilbige Wörter zu segmentieren und sich zu merken. Ähnliches wird für Sprachen mit starkem Wortakzent (stress) berichtet (für das Deutsche: z. B. Weber et al. 2004, für das Niederländische: z. B. Houston et al. 2000), nicht jedoch für Sprachen, denen diese Art Akzent fehlt (für das Französische: Nazzi et al. 2005).

Dieses frühe Sprachlernen führt von zuerst globalen akustischen Eigenschaften und Merkmalen des Sprachsignals zu immer spezifischeren Teilen der Sprachstruktur. Daher kann man bei diesem Vorgang von einem Gestaltprozess im Sinne Wertheimers $(1922,55)$ ausgehen als »[..] Erfassen bestimmter Ganzeigenschaften, Ganzbedingungen, Struktureigenschaften und von da aus der Weg zu >Teilen, im prägnanten Sinne dieses Wortes«. Mithilfe der »Faktoren« der Gleichheit, Nähe und des gemeinsamen Schicksals (Wertheimer 1923) und dank ihrer Fähigkeit, Vorkommenshäufigkeiten zu erkennen, organisieren und segmentieren Kinder das lautliche Signal perzeptiv in charakteristische Gestalten und Ganzheiten. Die Herausforderung im Laufe der Sprachaneignung besteht nun darin, dass sie den spezifisch sprachlichen Informationsgehalt dieser Gestalten erkennen müssen. Hilfreich ist ihnen dabei, wie ich nun zeigen möchte, die poetische Funktion von Sprache (Jakobson 1960, 1970).

In seinem Kommunikationsmodell beschreibt Jakobson (1960) sechs Funktionen einer sprachlichen Äußerung im Bezug auf verschiedene Aspekte des Kommunikationsprozesses (z. B. im Bezug auf den Sender, Empfänger, den Kontext usw.). Die poetische Funktion einer Äußerung ist ein Prozess, durch den die besondere sprachliche Beschaffenheit der Botschaft, »die Wörter und ihre Zusammensetzung, ihre Bedeutung, ihre äußere und innere Form " (Jakobson 1970, 20) in den Vordergrund gestellt, bzw. ins Bewusstsein gerückt werden. Dies geschieht über folgenden 
Mechanismus, den Jakobson $(1960,358)$ in seiner viel zitierten Definition beschreibt: "The poetic function projects the principle of equivalence from the axis of selection into the axis of combination. Equivalence is promoted to the constitutive device of the sequence." Mit dem Äquivalenzprinzip bezieht sich Jakobson auf morphosyntaktische und lexikalische Bedeutungsklassen, deren Einzelelemente aufgrund der Klassenzugehörigkeit zueinander in Beziehung stehen (äquivalent sind). In vorwiegend poetischen Äußerungen werden nun laut Jakobson weitere Äquivalenzen durch die prosodische Struktur gebildet: Es entstehen neue Klassen auf syntagmatischer Ebene aufgrund ähnlicher Vokale, gleicher Silbenreime, -länge, -betonung oder -position und anderen lautlichen Eigenschaften. »Meist ist die Poetizität lediglich Bestandteil einer komplizierten Struktur, doch ein Bestandteil, der die übrigen Elemente notwendigerweise verändert und die Beschaffenheit des Ganzen mitbestimmt "(Jakobson 1970, 20). Wie diese Veränderung vor sich geht, erläutert Posner $(1972,217)$ etwas genauer. Nach seinem Modell setzt sich der poetische Prozess zwischen den einzelnen morphologischen, syntaktischen oder semantischen Äquivalenzklassen vertikal fort, auch hier entstehen neue Verknüpfungen. An einem Beispiel aus dem Sonett "Les Chats" von Baudelaire verdeutlicht er dies: Die Wörter étincelles "Funken", parcelles d'or "Goldparzellen" und prunelles "Pupillen" stehen jeweils am Versende und bilden eine horizontale Äquivalenzklasse im Gedicht. Gleichzeitig entsteht eine vertikale Äquivalenzbeziehung zwischen der phonetischen Eigenschaft der Wörter, dass sie alle durch das Suffix (c)elles einen hellen Klang besitzen, und der semantischen Eigenschaft, dass sie etwas semantisch Helles oder Funkelndes bedeuten. Die Synästhesie führt zu einer vertikalen Äquivalenz zwischen der phonetisch-phonologischen und semantischen Ebene.

Mazzola $(2003,3177)$ ist überdies der Ansicht, dass die poetische Funktion sowohl musikalischen als auch sprachlichen Strukturen innewohnt und lässt an eine Parallele zwischen den Verarbeitungsmechanismen denken:

Together with horizontal Jakobson layers, these vertical Posner layers yield a standard construction not only for linguistic sign complexes, but also - even more prominently - for music. [...] This amounts to the thesis that musical meaning is construed in the vertical dimension from elementary ingredients of horizontal poetic structures.

Nichts anderes geschieht meines Erachtens beim frühen Spracherwerb, sofern die These des Prosodic Bootstrapping zutrifft. Kleinkinder bilden zunächst lautlichklangliche Äquivalenzklassen aus dem sprachlichen Input, auf denen dann weitere morphosyntaktische und lexikalische Äquivalenzbeziehungen aufgebaut werden können. ${ }^{2}$ Unter diesen Voraussetzungen kann der frühe Spracherwerb als Prozess beschrieben werden, der unter anderem auf der poetischen Funktion von Sprache

${ }^{2}$ Ebenfalls denkbar wäre, auf solchen Klassen im Sinne Mazzolas (2003) ein musikalisches System aufzubauen. Inwieweit Sprache die Ausprägung musikalischer Strukturen beeinflusst und umgekehrt, ist bislang jedoch nicht ausreichend untersucht. 
aufbaut. Er ist somit auch ein poetisch gerichteter Prozess, der möglicherweise eine starke musikalische Dimension im Sinne Mazzolas (ibid.) aufweist. Auch die Psychologin Sandra Trehub $(2003,14)$ formuliert dies, indem sie ein Kleinkind als "musical being« bezeichnet, dessen Wahrnehmung optimal auf die Verarbeitung akustischer Struktur ausgerichtet sei. Der Vorteil dieser Betrachtungsweise - also die Betonung der kindlichen Sprachwahrnehmung als Gestaltprozess und damit verbunden die gemeinsame Erfassung musikalischer und sprachlicher Strukturen unter dem Vorzeichen der poetischen Funktion - liegt darin, dass man einen anderen Blick auf das Spektrum an relevantem Input für Kinder im frühen Spracherwerb werfen kann. Sowohl gesprochene als auchgereimte und gesungene Sprache beinhalten die oben genannten Äquivalenzbeziehungen, die zu einer Optimierung poetischer Struktur und Gestalthaftigkeit führen können (s. Abschnitt 4). Zunächst werden jedoch die folgenden beiden Abschnitte skizzieren, welche Besonderheiten die akustische Struktur des gesprochenen Inputs aufweist, den Kleinkindern in den ersten Lebensmonaten erhalten und welche Reaktionen Kinder auf gesprochene und gesungene Sprache zeigen.

\section{Sprachlicher Input im frühen Spracherwerb}

Wie aus der Literatur bekannt ist, hat der an Kleinkinder bis zum Alter von 18 Monaten gerichtete sprachliche Input besondere Eigenschaften: Er ist vor allem prosodisch viel stärker strukturiert als Gespräche zwischen kompetenten erwachsenen Sprechern und erregt dadurch bei den kindlichen Adressaten größere Aufmerksamkeit. Diese Form gesprochener Sprache wird in der Literatur als Ammensprache, Motherese oder Babytalk bezeichnet. Sie besitzt allgemein folgende Merkmale: Erwachsene sprechen mit Kindern in höherer Sprechlage, nutzen einen größeren tonalen Umfang und auffällige Tonhöhenkonturen, sie artikulieren langsamer und klarer, betonen stärker, markieren die Grenzen syntaktischer Einheiten deutlicher und wiederholen häufiger (z. B. Ferguson 1977; Fernald et al. 1989; Stern et al. 1983; Fernald/Simon 1984; Bernstein-Ratner 1986; Kuhl et al. 1997; Chelibanova 2002; Burnham/Kitamura/Vollmer-Conna 2002; Englund/Behne 2006). Zudem ersetzen einige Eltern einzelne Laute oder lassen sie ausfallen (z. B. dt. ১bit du groß! statt ‘bist du groß!‘), konstruieren Sätze syntaktisch einfach, verwenden einen Spezialwortschatz (z. B. frz. >lolo` anstatt ’lait‘ für `Milch`) und viele phatische Füller (z.B dt. 'gell? `na?), die in ihrer Tonkontur stark überzeichnet sind. Diese Strukturmerkmale verändern sich mit dem Alter des Kindes nach dessen Bedürfnissen (z. B. Garnica 1977; Kitamura/Burnham 2003; Henning/ Striano/Lieven 2005). Schon Säuglinge reagieren in den ersten Wochen ihres Lebens stark auf dieses Sprachregister: Sie sind aufmerksamer, lächeln häufiger, fixieren ihren Gesprächspartner länger und versuchen ihre Körperbewegungen mit den Äußerungen zu synchronisieren. Zudem vokalisieren sie mehr, wenn sie auf diese 
Weise angesprochen werden (z. B. Condon/Sander 1974; Fernald/Kuhl 1987; Cruttenden 1994; Niwano/Sugai 2002; s. Beispiele unter Punkt 3). Motherese stimuliert Kleinkinder nicht nur zur Interaktion und steigert ihre Aufmerksamkeit, sie hilft offenbar auch bei der Sprachaneignung: Wie Thiessen/Hill/Saffran (2005) zeigten, erkannten sieben Monate alte Kinder dieselben Wörter im Textzusammenhang besser, wenn sie in Motherese gesprochen waren als wenn sie für Erwachsene gesprochen wurden. Eine Studie von Liu/Kuhl/Tsao (2003) ergab einen positiven Zusammenhang zwischen extremer Vokalartikulation in der Motherese und der Fähigkeit der Kinder, verschiedene Vokalqualitäten zu unterscheiden.

Die Motherese bildet jedoch nur einen Teil des sprachlichen Inputs, den Kinder erhalten. Gerade in den ersten drei Lebensjahren wird mit Kindern viel gesungen und gespielt, wobei häufig Reime zum Einsatz kommen. Dies belegt unter anderem eine in den Jahren 2005 und 2006 als Vorstudie zur Dissertation von Falk (2009) durchgeführte Befragung von 108 Eltern im Münchner Raum, deren Kinder bis zu zwei Jahre alt waren. Die Befragung fand in den Ambulanzen zweier Kinderkrankenhäuser, bei zwei Kinderärzten, in zwei Rückbildungskursen sowie in einer Krabbelgruppe statt. 93 Prozent der befragten Eltern gaben an, mit ihren Kindern zu singen, davon über die Hälfte (56 Prozent) sogar mehrmals täglich. Weitere 32 Prozent sangen immer noch mehrmals pro Woche. Insgesamt 75 Prozent der Eltern erklärten, dass sie mit ihren Kindern auch Spiele mit Reimen spielten, Reime aus Büchern vorlasen oder selbst kleine Reimereien erfanden. Diese Formen sprachlichen Inputs wurden in der Spracherwerbsforschung und in der Diskussion um den Einfluss der Motherese auf die Sprachentwicklung bisher kaum berücksichtigt. Allerdings wurde die musikalische Seite der Motherese bereits betont (z. B. Papoušek 1994). Weitere Arbeiten zeigen, dass sich kindgerichtetes Singen in ähnlicher Weise vom erwachsenengerichteten Singen abhebt wie kindgerichtetes Sprechen vom erwachsenengerichteten Sprechen: Eltern nutzen eine leicht erhöhte Tonlage und langsameres Tempo (z. B. Trehub/Unyk/Trainor 1993), sie artikulieren Vokale klarer, machen phonologische Kontraste deutlich und atmen konsistent an syntaktischen Einschnitten (Falk 2009). Säuglinge lassen sich vom Singen in Bann ziehen, bewegen sich weniger und sehen ihren Kommunikationspartner länger an (z. B. de l'Etoile 2006). Bereits zwei Monate alte Kinder stellen sich beim Singen auf ihren Kommunikationspartner ein. Wie Malloch (1999) zeigt, entwickelt sich eine Art rhythmisches Muster zwischen dem Gesang der Mutter und den Vokalisationen des Kindes. Mit zunehmendem Alter fordern Kinder von ihren Eltern direkt ein, dass sie mit ihnen singen sollen. Ein zweieinhalb Jahre altes Mädchen beispielsweise bringt seiner Mutter während des Nachmittagstees ein Buch mit folgender Bitte: "Mama, sing mir mal das Buch!« Dieses Zitat aus eigener Feldforschung zeugt von der Faszinationskraft des Singens und lässt vermuten, dass sprachliche Strukturen für Kinder durch die zusätzlichen musikalischen Merkmale interessanter, vielleicht sogar besser verständlich werden. 
3. Reaktionen von Kleinkindern auf Motherese und Gesang: Zwei Beispiele

Beim Wickeln finden gerade in den ersten Lebensmonaten besonders intensive Interaktionen zwischen Eltern und Kind statt. Das Beispiel einer russischen Mutter soll verdeutlichen, wie solche Gespräche das Kind sprachlich anregen. Die Mutter spricht in dieser kleinen Szene kindgerichtet, artikuliert sehr langsam und deutlich. Ihre Tochter, 12 Monate alt, spricht bisher nur wenige Silben und zwei Wörter, darunter $\gg$ Mama und `Papa . Die Mutter möchte sie dazu bringen, `Mama` zu sagen.

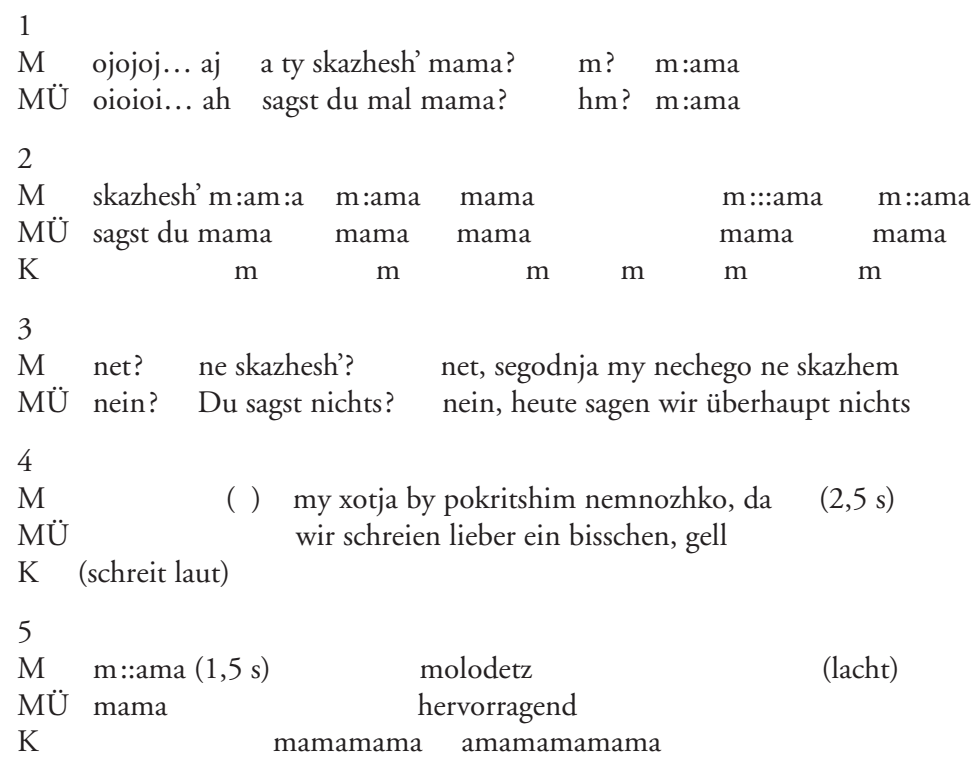

Transkript 1: Transliterierung der kyrillischen Buchstaben nach dem GOST-16876-71-System, die Transkription erfolgt in Partiturschreibweise nach vereinfachten HIAT-Konventionen, s. Ehlich/ Rehbein 1976. M: Mutter, MÜ: Übersetzung der Mutter, K: Kind; Doppelpunkte zeigen besonders lang artikulierte Laute an.

Hier ist gut zu sehen wie das Kind versucht, seine Produktion der Artikulation der Mutter anzupassen. Im Abschnitt 2 produziert es sechs Mal den Nasal `m $\mathrm{m}$ hintereinander während die Mutter das Wort 'mama vorspricht. Das Wort wird jedes Mal mit anderer Tonhöhenkontur gesprochen, was abwechslungsreich ist und Aufmerksamkeit erregt. Die Mutter hebt den Nasal besonders hervor, indem sie ihn stark dehnt, zudem ist durch den phatischen Füller 'm? im Abschnitt 1 noch ein weiterer Nasal vorhanden. Das Kind greift in der Folge diesen besonders prominenten Laut auf. Durch die direkte Face-to-Face-Situation kann es den Verschluss der Lippen bei seiner Mutter sehr gut beobachten. Die Artikulationen des Kindes sind fast rhythmisch zu nennen. Die Anfänge liegen etwa 1-1,3 Sekunden auseinander. In den ersten drei `Mamar-Sequenzen artikuliert die Mutter zu- 
erst, das Kind folgt, danach artikuliert das Kind zuerst, was wie ein wechselseitiges Imitieren des Nasals wirkt. Allerdings bleibt das gewünschte Ergebnis noch aus. Es scheint, als ob das Kind dies merkt und vor Wut laut aufschreit. Im Abschnitt 5 gelingt es dem Kind schließlich die Silbenfolge zu produzieren, was die Mutter sofort lobend bestätigt.

Wie im ersten Beispiel schon zu sehen war, reagieren Kleinkinder in ihren Produktionen oft verzögert, was verschleiert, dass sie das eben Gehörte tatsächlich verarbeiten. Moog (1963) nahm noch an, dass bis zum Alter von 18 Monaten keine Ähnlichkeiten zwischen Vorgesungenem und den Lautungen des Kindes besteht. Dass dies nicht immer so ist, soll das Beispiel in Abbildung 1 verdeutlichen.

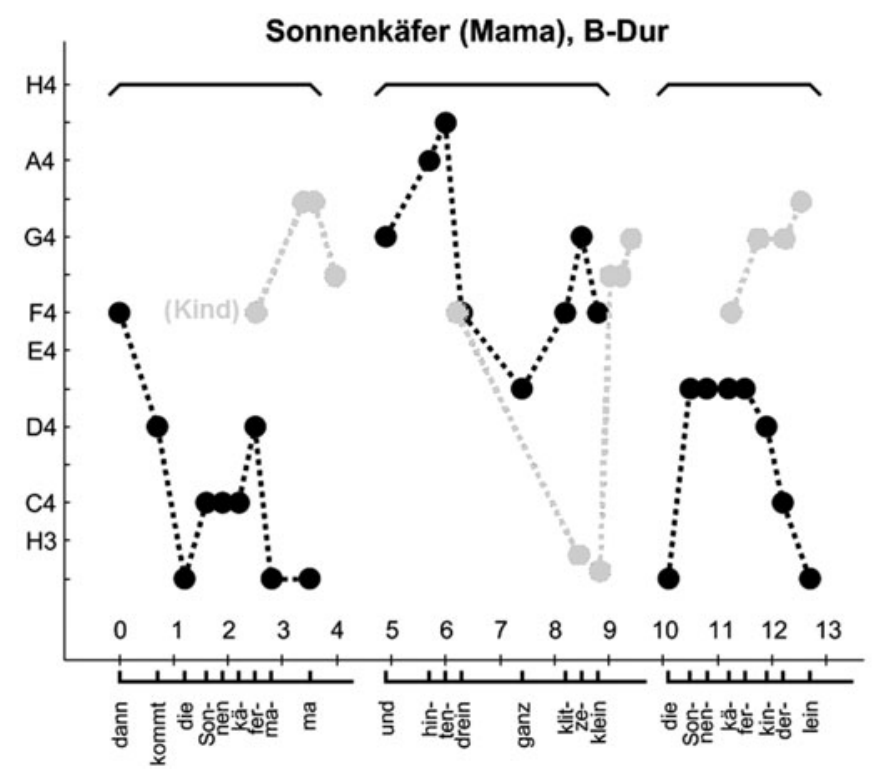

Abbildung 1: Synchrones Lauten des Kindes während die Mutter ein Kinderlied singt. Die y-Achse lokalisiert die Tonhöhen auf der Tonleiter, die x-Achse zeigt die Silben in ihrer zeitlichen Abfolge (in Sekunden) an. Punkte stellen diskrete Tonhöhen dar, Linien markieren zusammenhängende Phrasen, Klammern grenzen die Melodiephrasen der Mutter ab. Abbildung erzeugt mit den Programmen Pitch Analyzer und Notation Viewer von Franz Elmer, s. Stadler Elmer (2002).

Das elf Monate alte Mädchen versucht die Melodie der Mutter nachzuahmen, wobei es die Zunge lallend hin und her bewegt. Wie zu erkennen ist, greift es einzelne Melodiebewegungen der Mutter auf, allerdings etwas verzögert und überzeichnet. Es ist wahrscheinlich, dass während der Verarbeitung das aktuell Gehörte zusätzlich mit den eigenen Produktionen des Kindes interferiert, was die Gesamtkontur verändert. Diese beiden Beispiele sollen anschaulich machen, dass Kleinkinder stark auf Gesprochenes und Gesungenes reagieren und für sie auffällige Aspekte des Gehörten in die eigenen Produktionen integrieren. 


\section{Gute Gestalten in kindgerichteter Sprache: Optimierung poetischer Struktur}

Wenn die Sprachaneignung - wie in 1 begründet - poetisch gerichtet erfolgt, dann sollten Kinder um so eher Gestalten wahrnehmen und verarbeiten, je besser die poetische Struktur des Gehörten ist. Was zeichnet nun eine zum Zweck der Sprachaneignung optimierte poetische Struktur aus? Um dies zu beantworten, müssen wir noch einmal auf das zurückkommen, was bereits in Abschnitt 1 und 2 dargestellt wurde:

1) Grundvoraussetzung für poetische Struktur sind Äquivalenzen zwischen prosodischen/lautlichen Ereignissen/Strukturen im Input. Diese Ereignisse und Strukturen können unterschiedlichen Ebenen angehören: Sie können Lautund Lautkombinationen betreffen, die rhythmische oder Pausenstruktur, sie können aber auch tonaler und harmonischer Natur sein oder durch Stimmfärbung ausgedrückt werden.

2) Die lautlichen Äquivalenzen müssen Verbindungen zu lexikalischen, morphosyntaktischen oder Diskurs-Strukturen erlauben. Diese Verbindungen sollten regelhaft und systematisch sein, auch wenn sie keine direkte Abbildung prosodischer auf lexikalische oder morphosyntaktische Ereignisse und Strukturen darstellen, z. B. müssen lautlich/prosodisch prominente Ereignisse und Strukturen nicht zwangsläufig auch lexikalisch oder morphosyntaktisch prominent sein. Es sollte lediglich gewährleistet sein, dass es überhaupt eine systematische Verbindung zwischen den verschiedenen lautlichen und grammatisch-lexikalischen Äquivalenzklassen gibt.

Von optimierter poetischer Struktur werde ich sprechen, wenn zwei Bedingungen erfüllt sind:

a) Je rekurrenter die prosodischen Ereignisse und Strukturen vorkommen, desto besser wird die Chance, sie als Äquivalenzklasse aufzufassen, d. h. desto optimaler ist die Struktur in Bezug zur Wahrnehmungsleistung. Diese Forderung beruht auf den Ergebnissen der Spracherwerbsforschung, die zeigt, dass Kinder auf häufige Strukturen besonders achten (z. B. Saffran/Newport/Aslin 1996).

b) Die Ereignisse und Strukturen müssen auffällig, d.h. prominent genug sein, damit das Kind sie auch als Gestalten wahrnehmen kann und lernt. Dies kann - allgemein gesprochen - mit Mitteln der Gleichheit und des Kontrastes, der zeitlichen Nähe oder Entfernung, der Wiederholung und dadurch entstehenden Wahrscheinlichkeiten für gute Fortsetzungen/gemeinsames Schicksal erreicht werden. 
Ich werde im Folgenden darstellen, welche dieser Mittel in gesprochenen, gereimten und gesungenen kindgerichteten Äußerungen von den Eltern eingesetzt werden und wie dadurch mögliche lautliche Äquivalenzklassen mit Bezug zu grammatischlexikalischen Strukturen entstehen. Dies werde ich an spontanen Äußerungen von Eltern diskutieren, die mit ihren Kindern im Alter zwischen 0 und 14 Monaten interagieren. Diese Aufnahmen wurden im Rahmen einer umfangreichen Untersuchung zur Struktur kindgerichteten Singens (Falk 2009) mit 46 deutsch-, russisch- und französischsprachigen Familien erstellt. Es zeigt sich, dass es sich bei Motherese, Reimen und Gesang um ein Kontinuum handelt, das auf verschiedenen Ebenen eine Optimierung der poetischen Struktur darstellt und dadurch reichhaltig gute Gestalten bieten könnte, um den Spracherwerb zu fördern.

\subsection{Rhythmische und metrische Äquivalenzen}

Die ersten Beispiele stammen von einer russischen Mutter, die in der Kommunikation mit ihrem viereinhalb Monate alten Sohn sehr viele spontane Reimereien nutzt. Es entwickelt sich ein rhythmisches Muster, das dazu beitragen könnte, Wortgrenzen besser hervorzuheben. ${ }^{3}$

(1)

(a) oj

(b) ustal mal'chik ?

(b) ustal mal'chik popugaichik

(d) mal'chik, zaichik, popugaichik!
$\mathrm{Oh}$

müder Junge?

müder Junge, (mein) Papagaichen.

Junge, Häschen, Papagaichen!

In diesem Text geschieht durch das Suffix ı-chikı eine starke lautliche Homogenisierung der Endsilben der Substantive. >-chikı ist eine Diminutivendung maskuliner Substantive. Beim Wort 'mal'chik Junge ist diese Diminutivbedeutung nicht mehr semantisch transparent, jedoch durchaus bei den Tiernamen. Der Gebrauch der Verkleinerungsform wird im Laufe der Äußerung von der Mutter regelrecht dramatisiert. Sie artikuliert in Phrase 1d die Affrikate [t $f]$ und den Plosiv $[\mathrm{k}]$ in den Endsilben mit starkem Druck, so dass der Vokal dazwischen kaum noch hörbar ist. Dadurch treten die vorangehenden wortbetonten Vokale stärker hervor. Das Diminutivsuffix bildet die rechte Wortgrenze, die linke Grenze wird durch ein metrisches Muster deutlich, das in Phrase 1d auch tonal unterstrichen wird. Diese Phrase nimmt eine vierhebige, trochäische Form ${ }^{4}$ an - wie es bei dieser Mutter häufig in Gesprächssequenzen mit ihrem Sohn der Fall ist. Sie wird in langsamerem Tempo gesprochen mit rhythmischer und tonaler Kontur, die an einen gesungenen Vers erinnert.

\footnotetext{
${ }^{3}$ Jede Intonationsphrase wird in einer eigenen Zeile angegeben.

4 Zur Besonderheit des Viertakters vgl. Mellmann (dieser Band).
} 


$\begin{array}{lllllllll}\begin{array}{l}(2 \mathrm{~d}) \\ \mathrm{f}\end{array} & \mathrm{f} & \mathrm{g} & \mathrm{g} & - & \mathrm{h} & \mathrm{h} & \mathrm{c} & \mathrm{c} \\ 8 & 8 & 8 & 8 & 16 & 8 . & 16 & 8 & 8 \\ \text { mal'chik, } & \text { zaichik, } & & \text { popugaichik! }\end{array}$

Zur Notierung: Die obere Reihe stellt die ungefähren Tonhöhen dar. Der Strich kennzeichnet eine Pause. Darunter stehen die rhythmischen Werte (16: Sechzehntel 8: Achtel, 8. : punktierte Achtel, 4: Viertel, 2: Halbe). Haupt- und Nebenbetonungen sind fett gedruckt.

Wortanfänge und -ende sind in dieser lautlichen Einbettung gut und rekurrent markiert - der Wortanfang durch eine betonte Silbe, deren Vokal deutlich hervortritt, das Wortende durch das Diminutivsuffix, zusätzlich hilft die tonale Struktur. Die Tonhöhen folgen der trochäischen Fußstruktur der Äußerung: Jeweils ein Fuß erhält die gleiche Tonhöhe, zur nächsten betonten Silbe findet ein Tonhöhenwechsel statt. Die Grenze zum Wort 'popugaichikı ist durch die Pause und den Terzsprung von g zu h besonders deutlich. Insgesamt lässt sich hier von einer optimierten poetischen Struktur nach den oben genannten Bedingungen sprechen und zwar erfolgt die Optimierung vorwiegend nach dem Prinzip der Gleichheit lautlicher und prosodischer Struktur (Wertheimer, 1923). Zum einen wird im gesamten Abschnitt eine lautliche Äquivalenzklasse der >-chik«-Wörter zugänglich, die morphologisch gesehen alle Maskulina sind. Kempe/Brooks 2001 vermuten, dass die russischen Diminutivformen die Aneignung der Genusklassen der Substantive und damit ihrer Deklinationsparadigmen erleichtern. In den Phrasen dieses Beispiels könnte das Kind aufgrund der Endung folgende Wort-Gestalten segmentieren: sustalmal'chikı, 'popugaichik', 'mal'chikı, 'zaichikı. Mit zusätzlicher Erfahrung kann das Kind erkennen, dass eine Wortgrenze zwischen `ustal und `mal'chikı vorliegt. Diese wird prosodisch dadurch kenntlich, dass es zu einem Hebungsprall (stress clash) zwischen den beiden Wörtern kommt, d.h. zwei betonte Silben (‘ustal und 'mal'chikı) folgen unmittelbar aufeinander, was innerhalb eines russischen Wortes nicht möglich ist. Zum anderen bildet das trochäische Fußmuster eine Äquivalenzklasse. Prosodisch wird dies dadurch unterstrichen, dass in Vers 1d die einzelnen Füße nach dem Prinzip der Gleichheit durch gleiche Tonhöhe markiert werden. Da Russisch lernende Kinder im ersten Lebensjahr - wie Deutsch und Englisch lernende Kinder auch - wahrscheinlich über ein trochäisches Muster Wörter segmentieren (Jusczyk/Houston/Newsome 1999; Weber et al. 2004; Chukovskij 2000), kann hier von optimierter poetischer Struktur gesprochen werden.

In einem weiteren Beispiel derselben Mutter entwickelt sich ein ähnliches trochäisches Muster wie in Phrase 1d. Im Gegensatz dazu wird eine Optimierung poetischer Struktur aufgrund des Prinzips des Kontrastes (der Ungleichheit nach Wertheimer 1923) erreicht. Es handelt sich um eine Wickelszene: 
(2)
(a) poshli myt' popu
(b) pojdjom myt' popu?
(c) mama myla Filip popu
(d) mama moet Filip popu

Wollen wir mal den Popo waschen

Werden wir den Popo waschen?

Mama hat Philipp den Popo gewaschen.

Mama wäscht Philipp den Popo.

Dieses Beispiel enthält vier Intonationsphrasen, wobei das Wort 'popu in seiner Akkusativform das grenzbildende Element ist. Die Phrasen 2c und 2d weisen eine vierhebige trochäische Form wie in Beispiel 1d auf, doch diesmal sind die Betonungsverhältnisse auffällig durch starke tonale Auf- und Ab-Bewegungen kenntlich gemacht.

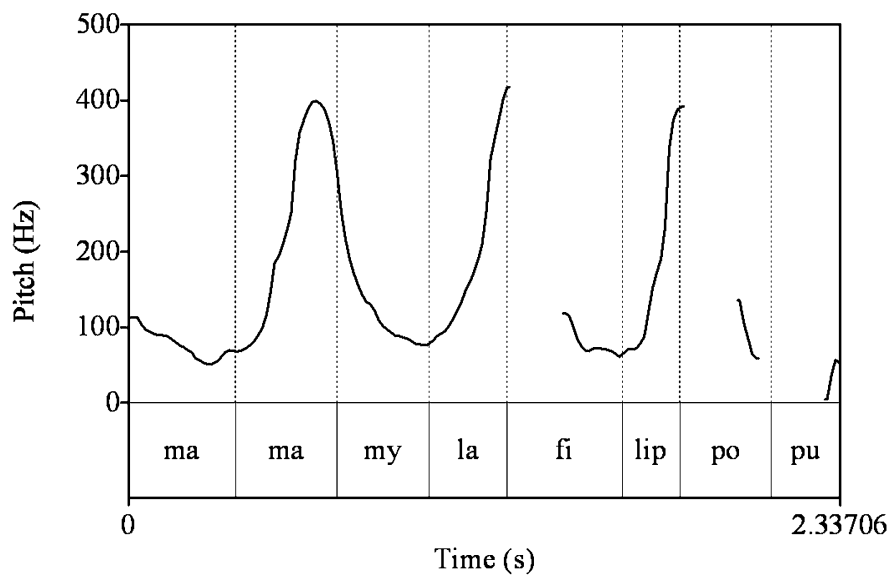

Abbildung 2: Frequenzkontur von Phrase 2c und Dauer der Silben. Frequenzanalyse erstellt mit Praat (Boersma 2001).

Die unbetonten Silben stehen bis auf die letzte Silbe der Phrase in großem tonalem Abstand zu den Akzentsilben. Dies ermöglicht einen besseren Kontrast zwischen betonten und unbetonten Silben. Zudem ist das Dauerverhältnis der betonten Vokale zu den unbetonten Vokalen auffällig: Betonte Vokale sind länger, in Phrase 2c haben sie sogar eine doppelt so lange zeitliche Ausdehnung wie unbetonte. Ein Wort ist in diesen beiden Phrasen als ein Kontrastmuster aus einem Tiefton auf einer betonten Silbe mit langem Vokal und einem Hochton auf einer unbetonten Silbe mit kurzem Vokal gut erkennbar - dies könnte Merkprozesse beim Kind erleichtern. Zusätzlich weist auch die globale tonale Kontur dieser Phrasen eine Besonderheit auf. Bei genauer Analyse zeigt sich, dass auf den Wortakzentsilben eine zwar vage, aber doch erkennbare Tonhöhenführung vorliegt.

(2c)

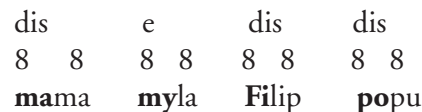


(2d)

$\begin{array}{lllllll}\text { dis } & & f & & g & & \text { dis } \\ 8 & 8 & 8 & 8 & 8 & 8 & 8 \\ \text { mama } & \text { moet } & \text { Filip } & \text { popu }\end{array}$

In diesem Beispiel zeichnet sich bereits das melodische und liedbildende Potential der Motherese ab, das im nächsten Abschnitt noch stärker in den Fokus rücken wird. Dabei werden die Wortakzentsilben durch tonale Stabilisierung in einen stärkeren Zusammenhang ${ }^{5}$ gebracht, was auch ein Verfahren sein könnte, um ihnen größere Prominenz zu verleihen.

\subsection{Melodische Äquivalenz}

Das nächste Beispiel zeichnet die Verfestigung eines Intonationsmusters nach. Dabei wird die Struktur zusehends musikalisiert, d.h. es deuten sich melodische und harmonische Prinzipien an, die eine weitere Äquivalenzebene aufbauen. Es handelt sich um eine Situation, in der eine deutschsprachige Mutter ihre fünf Monate alte Tochter wickeln will. Sie spricht sie folgendermaßen an:

$\begin{array}{lllll}\text { Kriegt } & \mathrm{H}^{*} & \mathrm{~L}^{*}+\mathrm{H} & \mathrm{L}^{*}+{ }^{\wedge} \mathrm{H} & \mathrm{L}^{*}+{ }^{\wedge} \mathrm{H} \\ \text { eine } & \text { neue } & \text { Windel? } & \mathrm{H}^{\wedge} \mathrm{H} \%\end{array}$

In diesem Beispiel sind die Tonakzente der Phrase in autosegmental-metrischer Notierung ${ }^{6}$ (GToBi, nach Reyelt et al. 1996) angegeben. Interessant ist das Syntagma reine neue Windek. Es weist drei Akzente auf, die intonatorisch einen Upstep (^) bilden, d. h. der jeweils folgende Akzent-, bzw. Hochton liegt auf einem höheren Tonhöhenniveau als der vorangehende (s. Abbildung 3).

So entsteht eine Art intonatorisches Treppenmuster, wobei die unbetonten Silben jeweils etwas höher liegen als die Akzentsilben, jedoch mit weniger Intensität gesprochen werden. Die Intonationsphrase endet auf einem hohen Grenzton, was einer Fragekontur in erwachsenengerichteter Sprache gleichkommt. Die Mutter wiederholt die Phrase (Bsp. 4), doch diesmal übersteigert sie das Intonationsmuster wie in Abbildung 4 (S. 244) zu sehen ist:

${ }^{5}$ Bregman (1990) hat in seinen Wahrnehmungsexperimenten mit Erwachsenen bereits gezeigt, dass solch eine Gruppierung perzeptiv prinzipiell möglich ist.

${ }^{6}$ Dies ist ein Transkriptionssystem für Intonation. H markiert einen Ton mit hohem Frequenzniveau, L einen Tiefton. Sterne $\left(^{*}\right)$ kennzeichnen Wortakzentsilben, die sogenannten Targets der Tonakzente. Ein Dach (^) steht für Upstep, $\mathrm{H}-{ }^{\wedge} \mathrm{H} \%$ symbolisiert einen hohen Grenzton am rechten Ende der Intonationsphrase. 


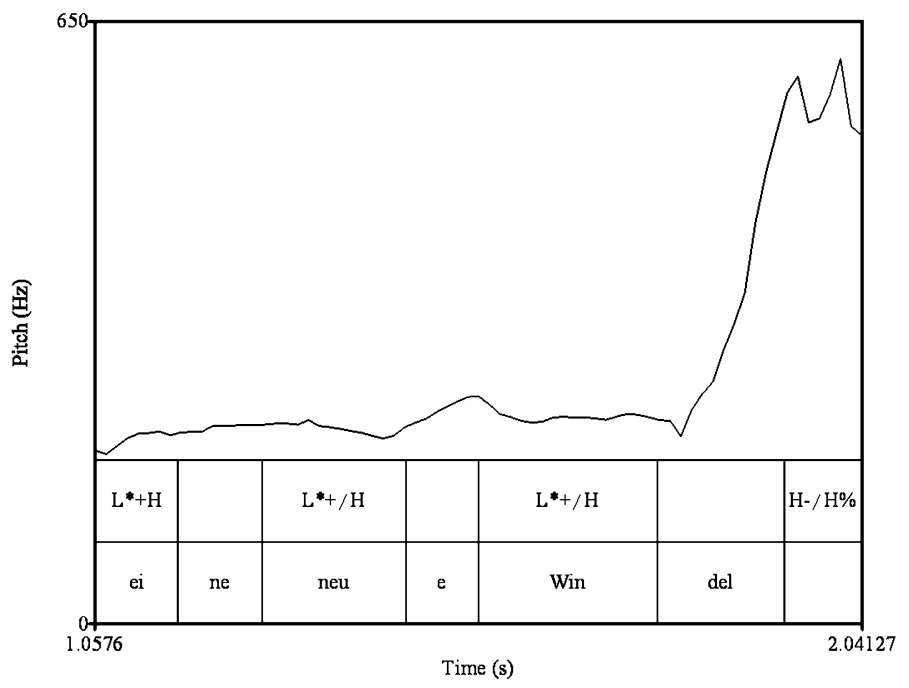

Abbildung 3: Frequenzkontur der Phrase seine neue Windel`, Tonakzente und Silben (erstellt mit Praat). Der Upstep wird mit dem Zeichen / wiedergegeben.

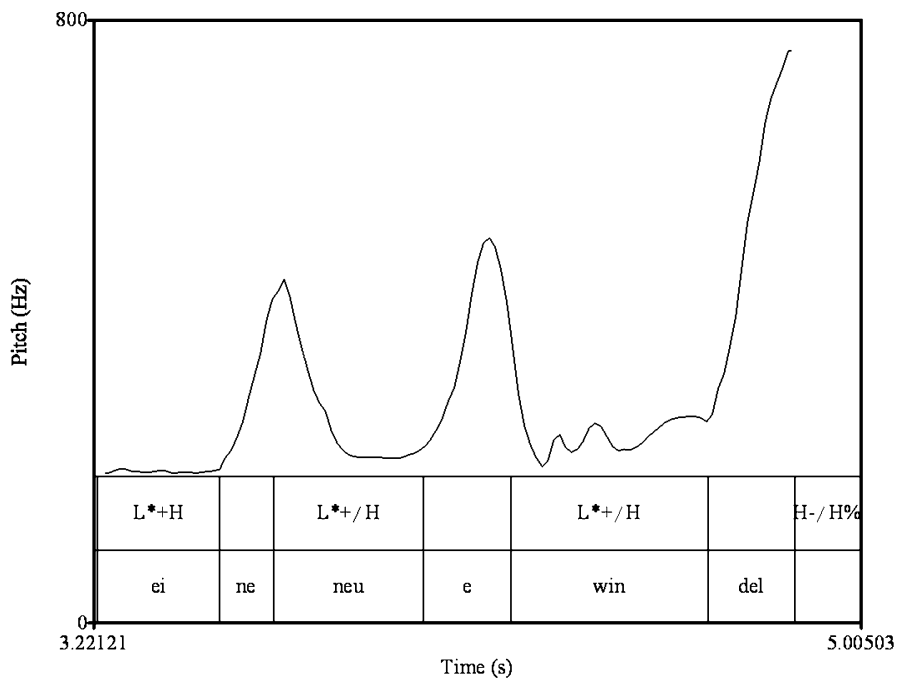

Abbildung 4: Stilisierte Frequenzkontur der Phrase reine neue Windel<, Tonakzente und Silben (erstellt mit Praat). Der Upstep wird in dieser Abbildung mit dem Zeichen / wiedergegeben. 
(4)

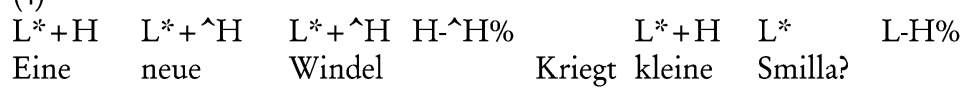

Daraus entwickelt sich ein kleines Lied ${ }^{7}$, das die Mutter zur Ankündigung des Wickelns singt.

\begin{tabular}{|c|c|c|c|c|c|c|}
\hline$g\left(g^{1}\right)$ & $a\left(a^{1}\right)$ & $d^{1}$ & $\mathrm{~h}$ & $\left(a^{1}\right)$ & $\mathrm{h}\left(\mathrm{h}^{1}\right)$ & $g^{1}$ \\
\hline 88 & 88 & 4 & 4 & 8 & 88 & 4 \\
\hline Eine & neue & Win & del & für die & kleine & Smil \\
\hline eine & neue & Win & del & kriegt die & kleine & Smil \\
\hline
\end{tabular}

Zunächst ist festzustellen, dass die Mutter die zweigeteilte Phrasenstruktur wie im Gesprochenen beibehält (seine neue Windel - für (kriegt) die kleine Smilla‘). Die Melodietöne sind tonal gut identifizierbar, sie gehören einer pentatonischen Leiter an. Der Upstep in der Intonationskontur der gesprochenen Phrase seine neue Windek wird hier zum Aufbau der melodischen Struktur verwendet. Die Töne der be-

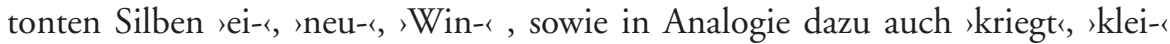
,Smil-^, bilden eine aufsteigende tonale Reihe. Die unbetonten Silben ı-ner, >-e`, `die und `-ner können als Durchgangstöne gelten, sie werden grob im Oktavabstand zu den vorangehenden Melodietönen gesetzt. Bemerkenswert ist auch die Veränderung, die gleichzeitig auf rhythmischer Ebene geschieht: Die zwei Teilphrasen werden von dieser Mutter als Viertakter gesungen. D.h. jede Phrase besteht aus insgesamt vier Zählzeiten und vier stabilen Melodietönen, in gesprochener Sprache waren hier jeweils drei (zwei) Tonakzente plus ein Grenzton vorhanden. Der Grenzton erfährt im Gesungenen die radikalste Veränderung: Er erhält eine eigene Zählzeit, was eine Verlangsamung am Phrasenende bewirkt und dadurch als guter Prädiktor der kommenden Grenze gelten darf (zum Konzept der phrasenfinalen Dehnung in Musik und Sprache, s. z. B. Lindbløm 1978). Zudem reiht sich das Phrasenende damit in typische Schlussklauseln von deutschen Kinderliedern (z. B. backe, backe Kuchen) ein.

Die gesungene Struktur spiegelt also wesentliche Aspekte der gesprochenen Struktur wider. Die Melodisierung der Intonation führt zu einer optimierten poetischen Struktur, denn die Abwandlungen, die die Mutter vornimmt, bringen Vorteile mit sich: Die Struktur wird im Sinne des Prinzips des gemeinsamen Schicksals/ der guten Fortsetzung regularisiert, durch die globale melodische und rhythmische Kontur bilden die beiden Teilphrasen zwei Äquivalenzklassen, die auch syntaktisch sinnvoll sind. Die Zugehörigkeit zu einer tonalen Reihe hebt die Wortakzentsilben als Äquivalenzklasse besonders hervor. Der Richtungswechsel der Melodie von

7 Die Notierung erfolgt wie in Beispiel 1, die in Klammern stehenden Tonhöhen können bis zu einem Ganzton von der Angabe abweichen. 
einer auf- zu einer absteigenden Linie auf den ersten Silben von 'Windel und sSmillar unterstreicht die beiden Wortanfänge. Diese stellen zentrale Bestandteile sowohl der syntaktischen Struktur (Köpfe der jeweiligen Phrasen) als auch der Diskurs-Struktur (Rhema, respektive Thema) dar. Insgesamt wird die Artikulation mit der prosodischen Stabilisierung langsamer: Während die Ausgangs-Intonationsphrase in der Motherese (Bsp. 3) lediglich 1.6 s dauert, ist schon die stilisierte Kontur (Bsp. 4) langsamer (2.5 s, enthält aber eine Pause von 0.8 s). Die gesungenen Phrasen (Bsp. 5) sind schließlich jeweils ca. 4.7 s lang. Diese Verlangsamung und die Wiederholung könnten die Verarbeitung des Gehörten sowie Erinnerungsprozesse erleichtern. Welche perzeptive Relevanz jeweils den Grenztönen im Gesprochenen und Gesungenen zukommt, ist noch unklar (Hinweise dazu in Dombrowski et al. 2007; Dombrowski 1995). Bemerkenswert erscheint zudem, dass die Stabilisierung der Intonationskontur hin zur Melodie sowohl in den russischen als auch in dem deutschen Beispiel über die Wortakzentsilben erfolgt, die die stärksten tonalen Bezüge untereinander haben. Die unbetonten Silben werden innerhalb der Phrase als Neben- bzw. Durchgangstöne arrangiert. Wie sieht es jedoch in einer Sprache wie dem Französischen aus, die im eigentlichen Sinne keine Wortakzentstruktur nutzt?

\subsection{Harmonische Äquivalenz}

In der folgenden Situation versucht eine französischsprachige Mutter die Aufmerksamkeit ihres neuneinhalb Monate alten Sohnes auf eine vorbeischleichende Katze zu lenken, deren Name `Mimosa lautet.

(6)
(a) Le chat!
(b) Il est où le chat?
(c) Il est où le chat, Clément?
(d) oui,
(e) c'est Mimosa
(f) Mimosa!

\author{
Übersetzung \\ Die Katze! \\ Wo ist sie, die Katze? \\ Wo ist sie, die Katze, Clément? \\ ja, \\ das ist Mimosa \\ Mimosa!
}

Die melodische Struktur dieser Phrasen lässt sich - bis auf das ıouiৎ, das eine steil aufsteigende tonale Bewegung auf nur einer Silbe aufweist - recht gut erkennen (die Notierung erfolgt wie in Beispiel 1).

$$
\begin{aligned}
& (6 a-c)
\end{aligned}
$$

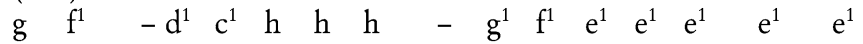

$$
\begin{aligned}
& \begin{array}{llllllllllllllll}
4 & 4 & 8 & 16 & 16 & 8 & 8 & 8 & 4 & 16 & 16 & 8 & 8 & 8 & 8 & 8
\end{array} \\
& \text { le chat il est où le chat il est où le chat Clément }
\end{aligned}
$$


In diesem Beispiel bildet sich aufgrund des harmonischen Zusammenhangs der Töne ein Muster, das diskursiv ausgenutzt wird. Durch den sehr deutlichen aufsteigenden Septimsprung auf ,le chat am Anfang wird eine Spannung aufgebaut, die sich bis zum Ende der Phrase $6 \mathrm{~b}$ fortsetzt, da die Töne sich weitgehend im Rahmen eines zu C-Dur gehörenden Dominantseptakkords bewegen. Die Auflösung erfolgt in Phrase 6c. Die tonale Spannung-Entspannung spiegelt sich im Aufmerksamkeitsprofil des Kindes: Mit den ersten beiden Phrasen versucht die Mutter, seine Aufmerksamkeit auf die Katze lenken, was ihr am Ende der dritten Phrase, in der tonal die Auflösung erfolgt, auch glückt. Dies wird sprachlich durch das folgende soui k klar. Die letzten beiden Phrasen 6e und f, die die Mutter nun mit der vollen Aufmerksamkeit des Kindes artikuliert, sind vom Ton und Rhythmus sehr verschieden. Die Artikulation ist deutlich verlangsamt, die Silben wirken im Vergleich zu den vorhergehenden Phrasen gedehnt. Die leichte tonale Spannung, die das langgezogene erste s-sa erzeugt, könnte wiederum dazu dienen, die Aufmerksamkeit des Kindes zu halten; dieses fällt am Ende der sechsten Phrase mit einem unartikulierten Laut in die letzte Silbe r-sar mit ein.

\begin{tabular}{|c|c|c|}
\hline (6e-f & & \\
\hline h & h g1 dis1 & h g1 (d1) \\
\hline 8 & 882 & 442 \\
\hline c'est & Mimosa & Mimosa \\
\hline
\end{tabular}

Die Struktur dieser Äußerung ist vor allem deswegen poetisch optimiert, da sich die einzelnen Tonhöhen der Silben - wie schon in den vorangehenden Phrasen 6a-d in einen harmonischen Rahmen einordnen und interpretieren lassen. Sie sind daher als harmonisch äquivalent anzusehen. Diese Äquivalenz wird zur Kommunikation bestimmter Diskursfunktionen ausgenutzt, in diesem Falle läuft die harmonische Spannung-Entspannung parallel zur Aufmerksamkeitssteuerung durch die Mutter. Gleichzeitig erfolgt die Melodisierung gleichmäßig über die Silben der Phrasen hinweg - im Gegensatz zum deutschen Beispiel 5, wo die Melodie in den Wortakzentsilben verankert war und eine trochäische Fußstruktur ausschlaggebend erschien.

Erwähnenswert an diesem Beispiel ist schließlich die rhythmische Struktur, da sie typisch für die französische Prosodie ist. Die rhythmische Struktur der ersten drei Phrasen hebt besonders die Silben schat, soù ı und >-ment hervor. schat sowie >-ment`stehen phrasenfinal vor einer Pause, soù hingegen wird langsamer gesprochen/gesungen als die beiden vorangehenden sehr schnell artikulierten Silben und ist deswegen prominenter. Phonologisch gesehen stehen die Silben jeweils am Ende einer groupe rhythmique - dies ist im Französischen ein Syntagma, das eine prosodische Einheit auf morphosyntaktischer Basis darstellt und auf der letzten Silbe einen Akzent erhalten kann, aber nicht muss (s. z. B. Mertens 1992). In diesem Fall sind alle potentiell betonbaren Silben hervorgehoben - durch die folgenden Pausen oder die Dauerverhältnisse zu anderen Silben. Die Gestaltung erfolgt 
aufgrund des Prinzips des Kontrastes der Silbendauern. Lange Silben markieren eine Äquivalenzklasse wichtiger Inhaltswörter, die gleichzeitig das Ende der französischen Redeeinheiten, der groupes rhythmiques, darstellen. Im russischen Beispiel 2 waren betonte und unbetonte Vokale ebenfalls in ihrer zeitlichen Ausdehnung kontrastiert, jedoch stilisierten die unterschiedlichen Dauern das metrische Fußmuster. Dies deutet an, dass sich die Optimierung poetischer Strukturen in kindgerichtetem Sprechen, Reimen und Singen im engen Rahmen der sprachspezifischen Prosodie abspielt.

\section{Zusammenfassung}

Die hier vorgestellten Äußerungen zeigen exemplarisch, wie sich im spontanen Sprechen mit Kindern prosodische Strukturen entwickeln, die musikalische und metrische Formen annehmen. Solche Strukturen wurden hier als poetisch optimiert bezeichnet, da sie geeignet erscheinen, einem spracherwerbenden Kind zu helfen, Laut-Gestalten im sprachlichen Input besser wahrzunehmen und sich daraus gemäß der Hypothese des Prosodic Bootstrapping - abstrakte sprachliche Formen anzueignen. Die Optimierung der poetischen Struktur entsteht in den dargestellten Fällen, indem die rhythmischen, metrischen oder tonalen (melodischen, harmonischen) Ebenen der Äußerung mit Hilfe der Gestalt-Faktoren der Gleichheit (Bsp. 1), bzw. des Kontrastes (Bsp. 2-5) und des gemeinsamen Schicksals (Bsp. 2, 5 u. 6) überstrukturiert werden und dadurch möglicherweise an perzeptiver Prominenz gewinnen.

Dies geschieht, wie in den Beispielen deutlich wurde, auf einzelsprachlicher prosodischer Grundlage. Die Ausnutzung der rhythmischen Struktur wie wir sie im Beispiel 6 gesehen haben, erscheint typisch für das Französische, wo die Dauer das verlässlichste Korrelat für einen Akzent darstellt. Im Gegensatz dazu haben wir in den russischen $(1,2)$ und deutschen $(3-5)$ Beispielen den Rückgriff auf ein metrisches fußbasiertes Muster gesehen, das tonal auf verschiedene Weise stilisiert wurde. Während im französischen Beispiel die tonale Struktur eher phrasenbezogen zugewiesen wurde, war sie im russischen und deutschen Fall an die Wortakzentsilben gekoppelt - auch dies weist auf die Verankerung in der jeweiligen prosodischen Struktur der Sprache hin.

Die prosodisch begründeten Äquivalenzklassen, die durch die Stilisierung bestimmter Merkmale in den vorgestellten Beispielen entstehen, verweisen auf lexikalische Funktionen (Wörter, Wortstämme), syntaktische Funktionen (Phrasen-, Wort-, Genusklassen) und Diskursfunktionen. Dadurch wird die in 4 aufgestellte Bedingung erfüllt, dass sich Verbindungen zwischen lautlich-prosodischen und anderen sprachlichen Ebenen herstellen lassen müssen, damit von poetisch optimierter Struktur gesprochen werden kann. Fazit der Überlegungen ist, dass es lohnenswert erscheint, sowohl gesungene als auch gereimte/metrisch gebundene Sprache in den Untersuchungen zur Motherese stärker zu berücksichtigen. Die tatsächliche per- 
zeptive Relevanz optimierter poetischer Strukturen und ihre Rolle im Spracherwerb sollte in zukünftiger Forschung genauer überprüft werden.

Simone Falk

Department I - Germanistik, Nordistik, Komparatistik

Ludwig Maximilians-Universität München

\section{Literatur}

Nan Bernstein-Ratner, Durational cues which mark clause boundaries in mother-child speech, Journal of Phonetics 14 (1986), 303-309.

Paul Boersma, Praat, a system for doing phonetics by computer, Glot International 5 (2001), $341-345$.

Albert S. Bregman, Auditory Scene Analysis. The Perceptual Organization of Sound. Cambridge, MA 1990.

Denis Burnham/Christine Kitamura/Ute Vollmer-Conna, What's new, pussycat? On talking to babies and animals, Science 296 (2002), 1435.

Olga Chelibanova, The influence of "Mother's speech « in the child's phonologic development during the first six month of life, in: Spanish Acoustical Society (SEA) (Hg.), Proceedings of the FORUM ACUSTICUM SEVILLA 16.-20. September 2002, Madrid 2002.

Kornej Chukovskij, Ot dvukh do pjati, Sankt-Petersburg 2000.

William S. Condon/Louis W. Sander, Neonate movement is synchronized with adult speech. Interactional participating and language acquisition, Science 183 (1974), 99-101.

Alan Cruttenden, Phonetic and prosodic aspects of Baby Talk, in: Claire Gallaway/Brian J. Richards (Hg.), Input and Interaction in Language Acquisition, Cambridge 1994, 135-152.

Ernst Dombrowski, Über strukturelle Gemeinsamkeiten zwischen sprachlichen und musikalischen Melodien, Jahrbuch der Deutschen Gesellschaft für Musikpsychologie 12 (1995), $110-133$.

Ernst Dombrowski/Thurid Holzrichter/Niels Münz/Alexander Nowak/Monika Poschmann, Prosodic rise and rise-fall contours and musical rising two-tone patterns, Proceedings of the XVI. ICPhS Conference, Saarbrücken (2007), 1245-1248.

Catherine H. Echols, Contributions of prosody to infants' segmentation and representation of speech, in: Jürgen Weissenborn/Barbara Höhle (Hg.), Approaches to Bootstrapping. Phonological, Lexical and Neurophysiological Aspects of Early Language Acquisition I, Amsterdam/ Philadelphia 2000, 26-46.

Catherine H. Echols/Megane Crowhurst/Jane B. Childers, The perception of rhythmic units in speech by infants and Adults, Journal of Memory and Language 36 (1997), 202-225.

Konrad Ehlich/Jochen Rehbein, Halbinterpretative Arbeitstranskription (HIAT), in: Linguistische Berichte 45 (1976), 21-41.

Kjellrun T. Englund/Dawn Behne, Changes in infant directed speech in the first six months, Infant and Child Development 15 (2006), 139-160.

Simone Falk, Musik und Sprachprosodie: Kindgerichtetes Singen im frühen Spracherwerb (Language, Context and Cognition), Berlin/New York 2009.

Charles A. Ferguson, Baby talk as a simplified register, in: Catherine E. Snow/Charles A. Ferguson (Hg.), Talking to Children, Cambridge 1977, 219-235. 
Anne Fernald/Patricia K. Kuhl, Acoustic determinants of infant preference for motherese speech, Infant Behavior and Development 10 (1987), 279-293.

Anne Fernald/Thomas Simon, Expanded intonation contours in mothers' speech to newborns, Developmental Psychology 20 (1984), 104-113.

Anne Fernald/Traute Taeschner/Judy Dunn/Mechthild Papoušek/Bénédicte de Boysson-Bardies/Ikuko Fukui, A cross-language study of prosodic modifications in mothers' and fathers' speech to preverbal infants, Journal of Child Language 16 (1989), 477-501.

Olga K. Garnica, Some prosodic and paralinguistic features of speech to young children, in: Catherine E. Snow/Charles A. Ferguson (Hg.), Talking to Children, Cambridge 1977, 63 88.

Lila R. Gleitman/Eric Wanner, Language acquisition. The state of the state of the art, in: Lila R. Gleitman/Eric Wanner (Hg.), Language Acquisition. The State of the Art. Cambridge 1982, 3-48.

Anne Henning/Tricia Striano/Elena V.M. Lieven, Maternal speech to infants at 1 and 3 months of age, Infant Behavior and Development 28 (2005), 519-536.

K. Hirsh-Pasek/D. Kemler-Nelson/P. W. Jusczyk/K. Cassidy/B. Druss/L. Kennedy, Clauses are perceptual units for young infants, Cognition 26 (1987), 269-286.

Derek M. Houston/ Peter W. Jusczyk/Cecile Kuijpers/Riet Coolen/Anne Cutler, Crosslanguage word segmentation by 9-months-old, Psychonomic Bulletin 7 (2000), 504-509.

Roman Jakobson, Linguistics and Poetics, in: Thomas Albert Sebeok (Hg.), Style in Language, New York/London 1960, 350-377.

-, Was ist Poesie?, in: ders., Poesie und Sprachstruktur. Zwei Grundsatzerklärungen [1933, 1934], Zürich 1970, 7-23.

Peter W. Jusczyk/Derek M. Houston/Mary Newsome, The beginnings of word segmentation in English-learning infants, Cognitive Psychology 39 (1999), 159-207.

Vera Kempe/Patricia J. Brooks, The role of diminutives in the acquisition of Russian gender. Can elements of child-directed speech aid in learning morphology?, Language Learning 51 (2001), 221-256.

Christine Kitamura/Denis Burnham, Pitch and communicative intent in mother's speech. Adjustments for age and sex in the first year, Infancy 4 (2003), 85-110.

Patricia K. Kuhl/Jean E. Andruski/Inna A. Chistovich/Ludmilla A. Chistovich/Elena V. Kozhevnikova/Viktoria L. Ryskina/Elvira I. Stolyarova/Ulla Sundberg/Francisco Lacerda, Cross-language analysis of phonetic units in language addressed to infants, Science 277 (1997), 684-686.

Shannon K. de l'Etoile, Infant behavioral responses to infant-directed singing and other maternal interactions, Infant Behavior \& Development 29 (2006), 456-470.

Björn Lindbløm, Final lengthening in speech and music, in: Eva Garding/Gösta Bruce/Robert Bannert (Hg.), Nordic Prosody. Papers from a Symposium, Lund 1978, 85-101.

Huei-Mei Liu/Patricia K. Kuhl/Feng-Ming Tsao, An association between mothers'speech clarity and infants' speech discrimination skills, Developmental Science 6 (2003), F1-F10.

Stephen N. Malloch, Mothers and infants and communicative musicality, in: Musicae Scientiae, Special Issue 1999/2000, 29-57.

Denise R. Mandel/Deborah G. Kemler-Nelson/Peter W. Jusczyk, Infants remember the order of words in a spoken sentence, Cognitive Development 11 (1996), 181-196.

Guerino Mazzola, Semiotic aspects of musicology. Semiotics of music, in: Roland Posner/ Klaus Robering/Thomas Albert Sebeok (Hg.), Semiotik. Ein Handbuch zu den zeichentheoretischen Grundlagen von Natur und Kultur (HSK, Band 13, 3), Berlin 2003, 3119-3188. 
Jacques Mehler/Peter W. Jusczyk/Ghislaine Lambertz/Nilofar Halsted/Josiane Bertonicini/ Claudine Amiel-Tison, A precursor of language acquisition in young infants, Cognition 29 (1988), $144-178$.

Katja Mellmann, Die metrische Gestalt. Mit Überlegungen zur Sinnfälligkeit des Viertakters, in: JLT 2:2 (2008), 253-272.

Piet Mertens, Intonation, in: Claire Blanche-Benveniste (Hg.), Le français parlé. Études grammaticales, Paris 1990, 159-176.

Helmut Moog, Beginn und erste Entwicklung des Musikerlebens im Kindesalter. Eine empirischpsychologische Untersuchung, Köln 1963.

James L. Morgan, A rhythmic bias in preverbal speech segmentation, Journal of Memory and Language 35 (1996), 666-688.

Thierry Nazzi/Laura C. Dilley/Ann Marie Jusczyk/Stefanie Shattuck-Hufnagel/Peter W. Jusczyk, English-learning infants' segmentation of verbs from fluent speech, Language and Speech 48 (2005), 279-298.

Thierry Nazzi/Franck Ramus, Perception and acquisition of linguistic rhythm by infants, Speech Communication 41 (2003), 233-244.

Marina Nespor/Irene Vogel, Prosodic Phonology, Dordrecht/Riverton 1986.

Katsuko Niwano/Kuniaki Sugai, Acoustic determinants eliciting Japanese infants' vocal response to maternal speech, Psychological Reports 90 (2002), 83-90.

Mechthild Papoušek, Melodies in caregivers' speech. A species-specific guidance towards language, Early Development and Parenting 3 (1994), 5-17.

Roland Posner, Strukturalismus in der Gedichtinterpretation, in: Heinz Blumensath (Hg.), Strukturalismus in der Literaturwissenschaft, Köln 1972, $202-242$.

Matthias Reyelt/Martine Grice/Ralf Benzmüller/Jörg Mayer/Anton Batliner, Prosodische Etikettierung des Deutschen mit ToBI, in: Dafydd Gibbon (Hg.), Natural Language and Speech Technology, Results of the third KONVENS conference, Bielefeld November 1996, Berlin/New York 1996, 144-155.

Jenny R. Saffran/Elissa L. Newport/Richard N. Aslin, Word segmentation. The role of distributional cues, Journal of Memory and Language 35 (1996), 606-621.

Elisabeth O. Selkirk, On Prosodic Structure and its Relation to Syntactic Structure, Bloomington, IN 1980.

Stefanie Stadler Elmer, Kinder singen Lieder. Über den Prozess der Kultivierung des vokalen Ausdrucks, Münster/New York/Berlin 2002.

Daniel N. Stern/Susan Spieker/R.K. Barnett/Kristine McKain, The prosody of maternal speech. Infant age and context related changes, Journal of Child Language 10 (1983), 1-15.

Erik D. Thiessen/Emily A. Hill/Jenny R. Saffran, Infant-directed speech facilitates word segmentation, Infancy 7 (2005), 53-71.

Sandra E. Trehub, Musical predispositions in infancy. An update, in: Isabelle Peretz/Robert Zatorre (Hg.), The Cognitive Neuroscience of Music, Oxford 2003, 3-16.

Sandra E. Trehub/Anna M. Unyk/Laurel Trainor, Adults identify infant-directed music across cultures, Infant Behavior and Development 16 (1993), 193-211.

Christiane Weber/Anja Hahne/Manuela Friedrich/Angela D. Friederici, Discrimination of word stress in early infant perception. Electrophysiological evidence, Cognitive Brain Research 28 (2004), 149-161.

Max Wertheimer, Untersuchungen zur Lehre von der Gestalt. I, Psychologische Forschung/Psychological Research 1 (1922), 47-58. 
-, Untersuchungen zur Lehre von der Gestalt. II, Psychologische Forschung/Psychological Rese$\operatorname{arch} 4$ (1923), $301-350$. 